\title{
Interstitial Electronic Localization
}

\author{
Bruno Rousseau* and N.W. Ashcroft \\ Laboratory of Atomic and Solid State Physics, \\ Cornell University, Ithaca, New York 14853-2501
}

(Dated: June 7, 2021)

\begin{abstract}
We investigate the ground-state properties of a collection of $N$ non-interacting electrons in a macroscopic volume $\Omega$ also containing a crystalline array of $N$ spheres of radius $r_{c}$ each taken as largely impenetrable to electrons and with proximity of neighboring excluding regions playing a key physical role. The sole parameter of this quantum system is the ratio $r_{c} / r_{s}$, where $r_{s}$ is the Wigner- Seitz radius. Two lattices (FCC and BCC) are selected to illustrate the behavior of the system as a function of $r_{c} / r_{s}$. As this ratio increases valence electrons localize in the interstitial regions and the relative band-width $\epsilon_{F} / \epsilon_{F}^{0}$ is found to decrease monotonically for both. The system is motivated by the behavior of the alkali metals at significant compression. It accounts for band narrowing, leads to electronic densities with interstitially centered maxima, and can be taken as a model which clearly may be improved upon by perturbation and other methods.

PACS numbers: 71.23.An,71.20.Dg,71.10.Ca
\end{abstract}


Wigner and Seitz [1] 2] originally observed that the major physical properties of sodium at one atmosphere $\left(10^{5} \mathrm{~Pa}\right)$ could be accounted for by considering the crystal as composed of two components, namely the ion cores, constituted by the nuclei and their bound electrons, and itinerant valence electrons, assumed statistically separate from the cores and in a paramagnetic state. For the stabilizing volume they found that the cores exerted only a weak perturbation on the valence electrons (leading to the nearly free electron (NFE) approximation) the core then occupying a relatively small fraction of the unit cell. As a starting point for the theory of the metallic state under ordinary conditions, the Wigner and Seitz description has been very successful over the years. However, high pressure experiments conducted recently are now challenging this point of view: when the cores are induced to occupy an increasingly larger fraction of the unit cell the indications are that a new paradigm may be appropriate, as is suggested here.

According to system, pressure can reduce linear dimensions by as much as $50 \%$ and under these conditions the alkalis depart notably from their expected "simple metal" (or NFE) behaviors [3]. Their crystal structures at room temperature generally proceed from BCC to FCC, and then to non-close packed (for a concise exposition of the structures adopted by the alkalis, see [4] and references therein). The latter are difficult to understand intuitively within the simple NFE and nuclear-centric viewpoint of Wigner and Seitz. Theoretical work also indicates a breakdown of the NFE model: Neaton et al. [5] [6], among others, reported $a b$ initio calculations for the band structure of lithium and sodium at high compression. As they showed, the bands are far from NFE-like and the occupied band-width is much smaller than the familiar 50.1/ $r_{s}^{2} \mathrm{eV}$ expected for free electrons. They also noted [6] that the combined effects of Coulomb repulsion, Pauli exclusion, and orthogonality result in an increase of valence electron density in the interstitial regions the valence electrons evidently being forced away from the near core regions characteristic of nuclear-centric electron distributions.

This behavior is shown to follow from a simple but quite different physical model which focusses on the increasing role played by core exclusion (and near neighbor core proximity) upon systematic increase of density, at least at energies typified by Fermi energies. First, consider a system of $N$ valence electrons and $N$ spheres (the "ions") occupying a common volume $\Omega$. Though many of the points to be made are general, the nature of the problem is illustrated with two simple lattices, here cubic Bravais lattices (BCC and FCC), with sites $\{\mathbf{R}\}$, and the cores will ultimately be taken to rigorously exclude the valence electrons. As 


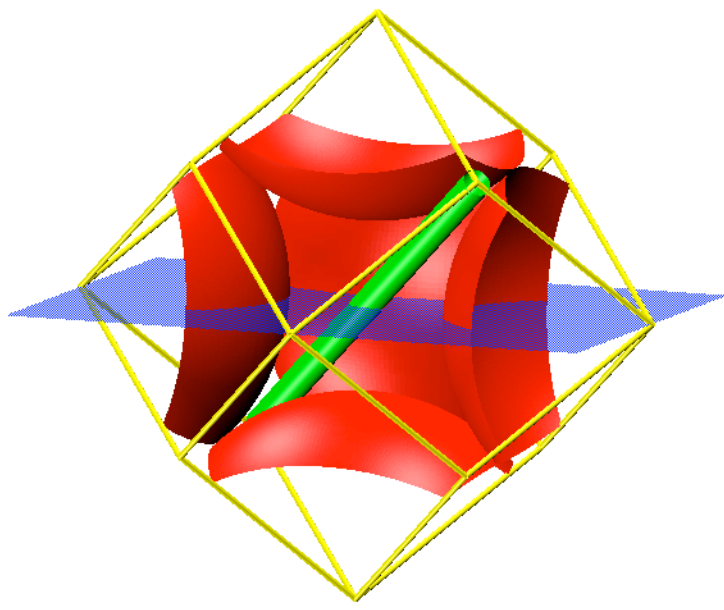

(a)FCC

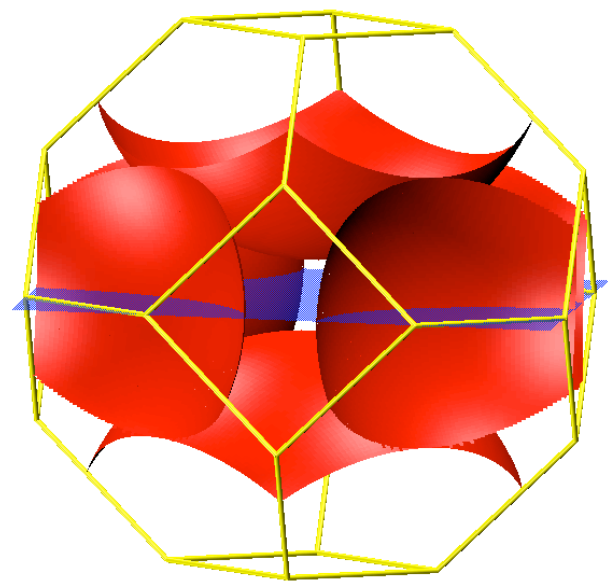

(b)BCC

FIG. 1: Interstitial Wigner Seitz cells. The red surfaces represent excluding spheres at close packing (one sphere is removed in the FCC cell for visibility). Indicated in blue is the plane in which the density for Figure 3 is computed and the green cylinder in the FCC cell represents the domain for Figure 2 .

in the formulation of the NFE model, electrons are also initially taken as non-interacting, though correlation effects will be of later significance. We consider the thermodynamic limit $N \rightarrow \infty, \Omega \rightarrow \infty, \Omega / N=4 \pi r_{s}^{3} a_{0}^{3} / 3$ and use periodic Von Kármán boundary conditions on $\partial \Omega$. As a starting point, the ion-electron interaction $v$ is modeled as (in Hartrees):

$$
v(\mathbf{r})=V_{0} \Theta\left(r_{c}-\frac{r}{a_{0}}\right) .
$$

where $a_{0}$ is the Bohr radius, $\Theta$ is the Heaviside function, $r_{c} a_{0}$ is the ionic radius and $V_{0}$ reflects how strongly excluding the potential is. The periodic non-interacting electronic Hamiltonian is thus:

$$
\hat{h}_{e}(\mathbf{r})=-\frac{\hbar^{2}}{2 m_{e}} \nabla^{2}+\sum_{\mathbf{R}} v(\mathbf{r}-\mathbf{R}),
$$

for which the Schrödinger equation is

$$
\hat{h}_{e}(\mathbf{r}) \psi_{i}(\mathbf{r})=\epsilon_{i} \psi_{i}(\mathbf{r})
$$

(a paramagnetic ground state is again initially assumed). As with the NFE approach Bloch's theorem fixes the general form of solution of (3). Using scaled variables:

$$
\bar{\Omega}=\frac{\Omega}{\left(r_{s} a_{0}\right)^{3}}, \quad \mathbf{X}=\frac{\mathbf{R}}{r_{s} a_{0}}, \quad \mathbf{x}=\frac{\mathbf{r}}{r_{s} a_{0}}
$$




$$
\bar{\nabla}=r_{s} a_{0} \nabla, \quad \bar{V}_{0}\left(r_{s}\right)=r_{s}^{2} V_{0}, \bar{r}_{c}=\frac{r_{c}}{r_{s}},
$$

the dimensionless form of (2) is:

$$
\bar{h}_{e}(\mathbf{x})=-\frac{1}{2} \bar{\nabla}^{2}+\sum_{\mathbf{X}} \bar{V}_{0}\left(r_{s}\right) \Theta\left(\bar{r}_{c}-|\mathbf{x}-\mathbf{X}|\right) .
$$

For fixed $\bar{r}_{c}=r_{c} / r_{s}$, two values of $V_{0}$ lead to renormalized eigenvalue equations independent of $r_{s} ; V_{0}=0$ and $V_{0} \rightarrow \infty$, the latter case related to the Quantum Lorentz Gas with a periodic configuration of scatterers [7]. The classical limit will have links to certain billiards problems.

The two-dimensional equivalent yields interesting insight into the problem ahead. Consider a hexagonal lattice of discs also taken as largely impenetrable to the electrons, as may be obtained by considering a (111) lattice plane either for BCC or FCC. At the limiting packing fraction in the plane of $\pi / 2 \sqrt{3}$, the circular regions touch, and per unit cell there remain two involuted triangular regions either of which must eventually contain a single electron leading to discrete but highly degenerate and eventually polar ground states. These triangular regions are essentially isolated and we know the band-width must vanish at close packing in this case. As the radius of the discs approach their close packing value, the triangular regions are connected by narrow windows and finite band-widths must result. The proximity of excluding discs (and their associated boundary conditions) will tend to heavily suppress the electronic density at these windows, making it more favorable energetically for the density to accumulate at the center of a triangular region. The band-width is expected to decay rapidly to zero as the radius of the discs approach their close packing value. Calculations carried out using the method described next show that this is exactly so. In three dimensions, it is not possible to fully isolate an interstitial volume, even at close packing; however, we expect the same argument to be valid, ie as the length scale associated with the windows becomes much smaller than the length scale associated with the inner region of the interstitial Wigner Seitz (IWS) cell, the band-width should decrease rapidly.

The associated eigenvalue problem can be formulated using a plane wave basis. For set $\{\mathbf{K}\}$, the reciprocal lattice, $\mathbf{k}$ a point in the first Brillouin Zone (1BZ), and $n$ a band index, the eigenvalue problem proceeds from:

$$
\begin{aligned}
\left\langle\mathbf{r} \mid \psi_{n \mathbf{k}}\right\rangle & \equiv \frac{1}{\sqrt{\Omega}} \sum_{\mathbf{K}} e^{i(\mathbf{K}+\mathbf{k}) \cdot \mathbf{r}_{c_{n}}(\mathbf{k})_{\mathbf{K}},} \\
h_{e}(\mathbf{k})_{\mathbf{K}, \mathbf{K}^{\prime}} & \equiv\left\langle\mathbf{K}+\mathbf{k}\left|\hat{h}_{e}\right| \mathbf{K}^{\prime}+\mathbf{k}\right\rangle,
\end{aligned}
$$




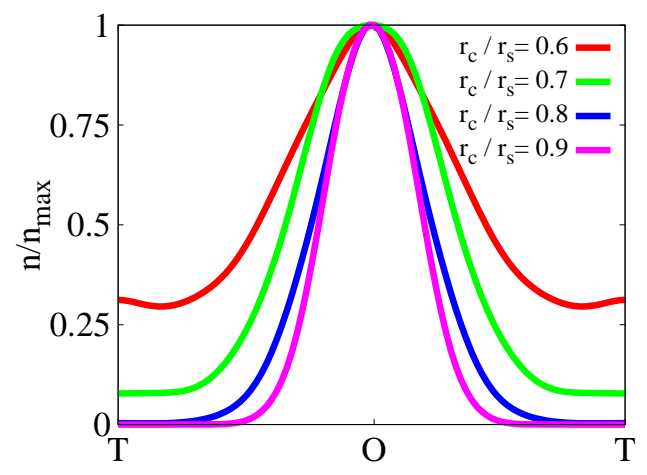

FIG. 2: Electronic density along the main body diagonal of the IWS cell of the FCC lattice for various values of the ratio $r_{c} / r_{s}$. The density is normalized to its maximum value at $\mathrm{O}$ for comparison purposes. $\mathrm{T}$ and $\mathrm{O}$ are the tetrahedral and octahedral sites.

yielding the standard

$$
\epsilon_{n \mathbf{k}} c_{n}(\mathbf{k}) \mathbf{K}=\sum_{\mathbf{K}^{\prime}} h_{e}(\mathbf{k}) \mathbf{K}, \mathbf{K}^{\prime} c_{n}(\mathbf{k})_{\mathbf{K}^{\prime}}
$$

For finite $V_{0}$, we consider an initial finite plane wave expansion. Reciprocal lattice vectors $\mathbf{K}$ are included in complete stars, all having a radius smaller than a cutoff $K_{\Lambda}$. Sums on the 1BZ are carried out using the special point technique [8] [9].

The formal limit $V_{0} \rightarrow \infty$ must eventually be considered as a boundary condition on the electronic orbitals, by demanding that they vanish on the surfaces of the excluding spheres. Implementing such a condition completely is quite complex and we use an approximate alternative: in the limit of large values of $V_{0}$, as is physically clear, the results become insensitive to the value of $V_{0}$ and thus approximate the limit $V_{0} \rightarrow \infty$. Convergence can be assessed by computing the lowest eigenvalue at $\Gamma$ (the center of the 1BZ), at special points on the surface of the $1 \mathrm{BZ}$ (H,N,P for the BCC lattice, X,W,L,K for the FCC lattice) and at points $\mathbf{k}$ pointing towards these special points and of magnitude $|\mathbf{k}|=k_{F}^{0}$ (the free electron Fermi wave vector). The parameters $V_{0}$ and $K_{\Lambda}$ were chosen to produce reasonable convergence with moderate computational effort[10].

From the solution for the states (6) we determined the electronic density, centering the Wigner Seitz cell around an interstitial site (defining an IWS cell, see Figure 1). In the coordinates of the standard cell the octahedral and the tetrahedral points of the FCC lattice have coordinates $\left(\frac{1}{2}, \frac{1}{2}, \frac{1}{2}\right)$ and $\left(\frac{1}{4}, \frac{1}{4}, \frac{1}{4}\right)$ and the interstitial site of the BCC lattice has coor- 


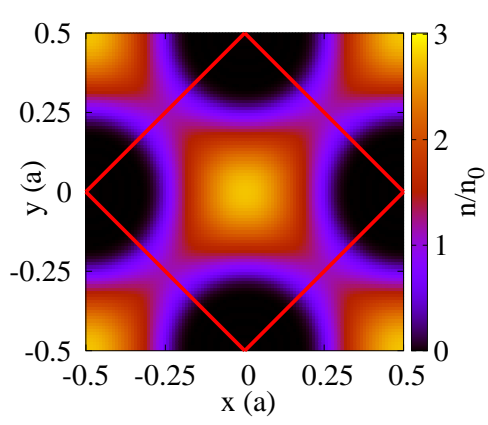

(a)FCC: $r_{c} / r_{s}=0.5$

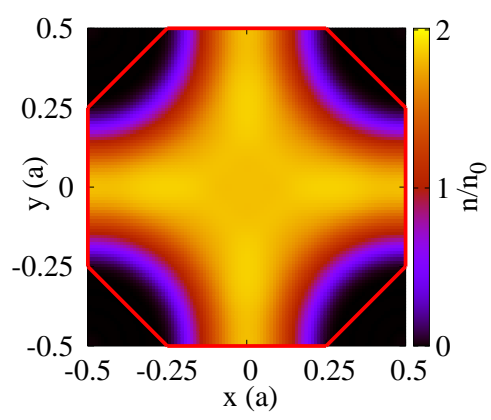

(c)BCC: $r_{c} / r_{s}=0.5$

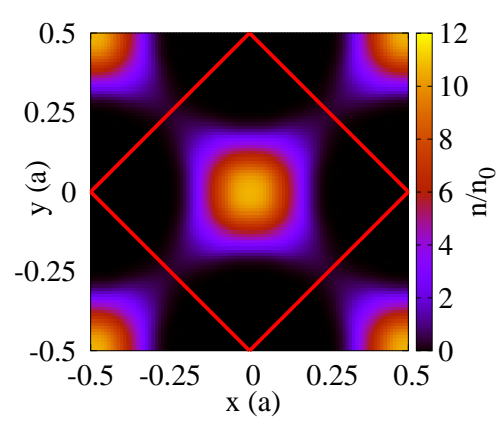

(b)FCC: $r_{c} / r_{s}=0.7$

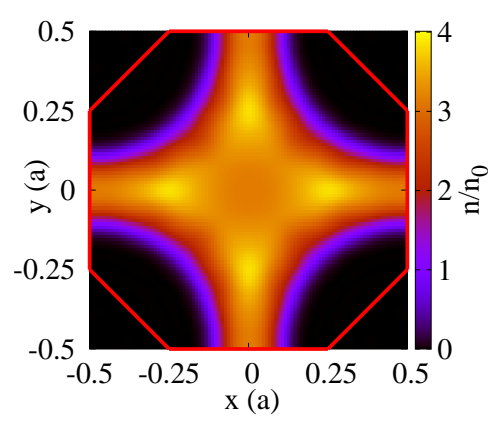

(d)BCC: $r_{c} / r_{s}=0.7$

FIG. 3: Electronic density (from (3)) in the $z=0$ plane. The intersection between the IWS cell and the $\mathrm{z}=0$ plane is outlined in red. Note that on the approach to close packing, the electronic density develops maxima at positions in the IWS cell that are maximally distant from the neighboring spheres.

dinates $\left(0,0, \frac{1}{2}\right)$ (these choices are not, of course, unique). The density has been computed on a plane of constant $\mathrm{z}$ value centered on the interstitial sites (Figure 3), and also along the body diagonal of the FCC IWS cell, from tetrahedral to octahedral to tetrahedral site (Figure 2). It clearly has a localized character and this provides insight into the different behavior seen for the two lattices as $r_{c} / r_{s}$ approaches close packing. As spheres take up increasing space in the FCC lattice, the only paths linking cavities centered at the octahedral sites of neighboring IWS cells must go through their shared tetrahedral site. As can be seen in Figure 2, the boundary conditions efficiently suppress the density at the tetrahedral site as $r_{c} / r_{s}$ approaches close packing, thus effectively turning the inner surface of the FCC IWS cell (ie the surface of the Wigner Seitz cell outside the spheres) into a surface where density vanishes. Boundary conditions which impose a vanishing of the electronic density at the 


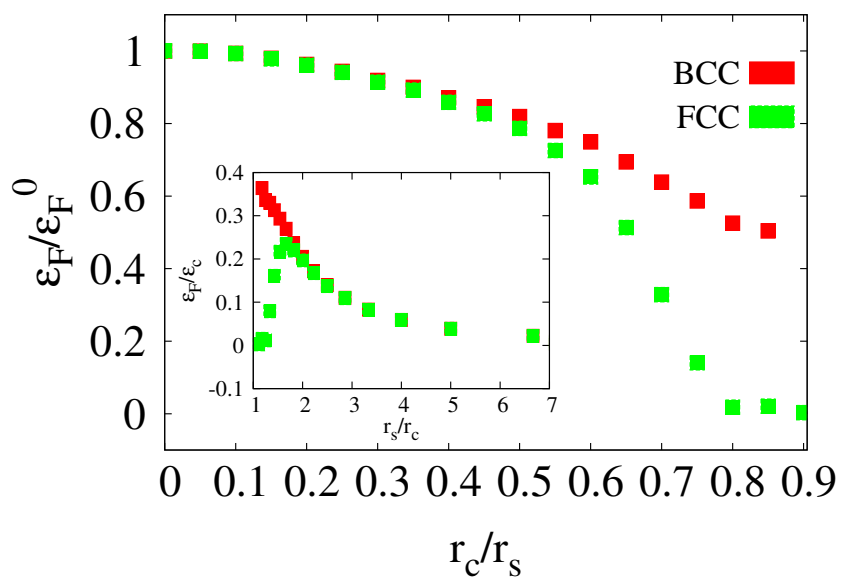

FIG. 4: Computed band-width normalized to the free electron Fermi energy as a function of the ratio $r_{c} / r_{s}$. Inset: absolute band-width, normalized to $\epsilon_{c}=\epsilon_{F}^{0}\left(r_{s}=r_{c}\right)$. We observe that the absolute band-width of the FCC lattice has a turnover around $r_{s} \simeq 2 r_{c}$, whereas for the BCC case it does not.

surface of the cell imply flat bands. The density at the surface of the IWS cell of the BCC lattice, on the other hand, is not as dramatically suppressed even at close packing, allowing for a significant band-width.

Bands and band-widths have been computed in both the FCC and BCC lattices and representative results can be seen in Figures 4 and 5. Relative band-widths decrease monotonically from the free electron value at $r_{c} / r_{s}=0$ (which we take to yield the empty-lattice bands) to a finite value in the BCC case and to low values in the FCC case at close packing $\left(r_{c} / r_{s} \simeq 0.905\right.$ for $\mathrm{FCC}$ and $r_{c} / r_{s} \simeq 0.879$ for BCC $)$. Furthermore, it appears that the Fermi surface develops necks at the L points of the BCC 1BZ, and almost so at $N$ in the FCC 1BZ[11]. In order to further substantiate these results, we also computed the eigenvalues (in units of $\epsilon_{F}^{0}$ ) at $\Gamma$ and at $\mathrm{H}$ for the BCC lattice using an expansion based on Kubic harmonics[12]. We determined the lowest eigenvalue at $\Gamma$ in the $\alpha$ representation and the lowest eigenvalues at $\mathrm{H}$ in the $\gamma$ and $\alpha$ representations[13]. The difference between the $\gamma$ eigenvalue at $\mathrm{H}$ and the $\alpha$ eigenvalue at $\Gamma$ decreases for $0 \leq r_{c} / r_{s} \leq 0.6$, suggesting strongly that the relative Fermi energy (the band-width) must follow suit. The method produced qualitatively different results according to the number of basis functions used at larger ratios, suggesting that the Bethe Von de Lage method, which tacitly assumes a nuclear-centric 


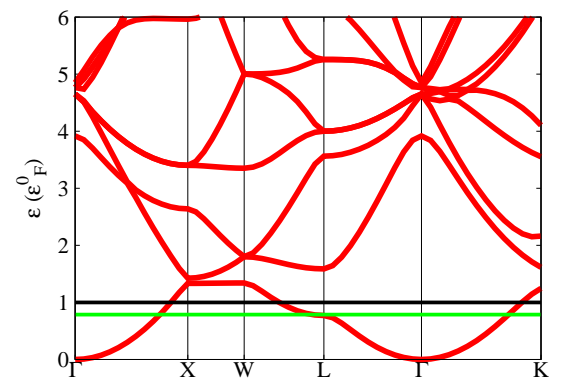

(a)FCC: $r_{c} / r_{s}=0.5$

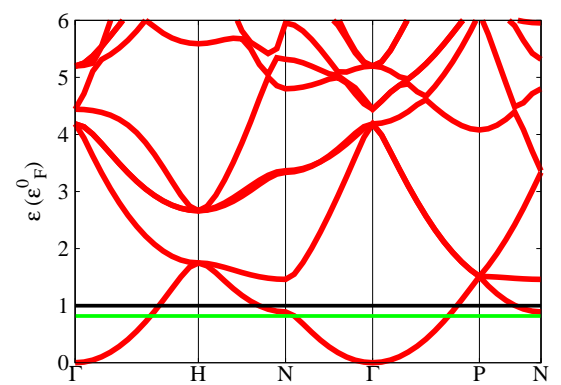

(c)BCC: $r_{c} / r_{s}=0.5$

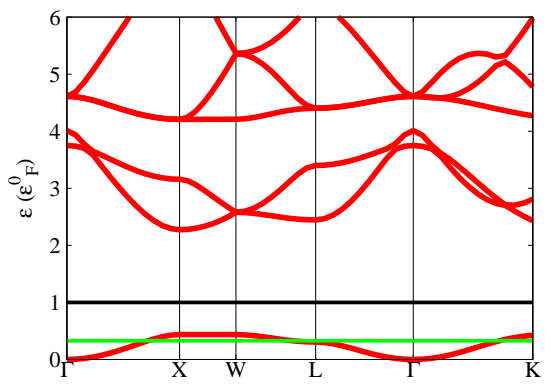

(b)FCC: $r_{c} / r_{s}=0.7$

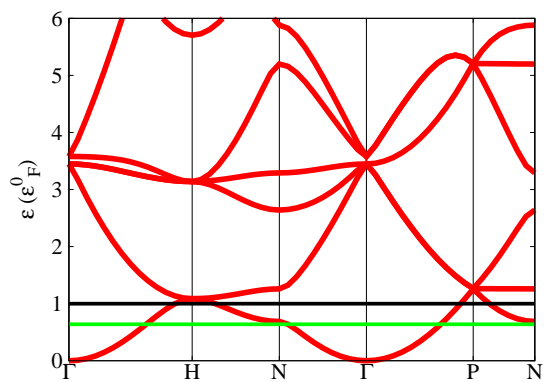

(d)BCC: $r_{c} / r_{s}=0.7$

FIG. 5: Eigenvalue spectrum for a few values of $r_{c} / r_{s}$ for excluding spheres in the FCC and BCC lattices. Energies are given in units of $\epsilon_{F}^{0}$, the free electron Fermi energy, and the energy at the bottom of the lowest band is set to zero. The Fermi energy and the free electron Fermi energy (1 in the graphs' units) are indicated.

view, does not converge quickly as the ratio approaches close packing.

The model simulates core exclusion in the simplest way and proceeds from weak coupling, NFE like behavior at small relative core volume to narrowing relative band-width (and narrowing absolute band-width for FCC) at large relative core volume. The rate at which the bands narrow depends on the connectedness of the IWS cell of the lattice and is expected therefore to be a general feature. The model reproduces qualitative features observed by others [5] 6], such as the narrowing of the band-width and strong modulations of the electronic density leading to interstitial site maxima. It also provides insight into the seemingly strange behavior observed earlier and is suggested as a possible initiating paradigm for the alkalis under high pressure which may serve as an alternative to the NFE approach. The ionic cores are not of course rigorously excluding (as is known from the existence of penetrating 
states), however the features of the model should partially subsist so long as the average kinetic energy of the valence electrons does not exceed the excluding core potential. In that sense, it provides insight into ab initio calculations using valence one pseudopotentials with repulsive ionic cores. It is unlikely however that the physical system would be well described by pseudopotentials designed only to reproduce zero pressure properties when the ionic cores are brought to the point of close packing.

For the physical situation of interest here it becomes clear that the electrons distribute themselves in a manner quite different from what the NFE limit would suggest. This can be deduced by invoking the simplifying features of the Wigner-Seitz approach, but for the largely confining volumes, themselves initially regarded as spheres. As a first approximation these have radii $\bar{r}_{s}=r_{s}\left\{1-\left(r_{c} / r_{s}\right)^{3}\right\}^{\frac{1}{3}}$ on the surfaces of which the wave-functions will vanish. The corresponding density is proportional to $\left\{\sin \pi\left(r / a_{0} \bar{r}_{s}\right) / \pi\left(r / a_{0} \bar{r}_{s}\right)\right\}^{2}$ and gives, near close packing, a quite accurate accounting of the density of Figure2, which follows from wave-functions (6). The corresponding scale of kinetic energy, $\left(\pi / \bar{r}_{s}\right)^{2} / 2$ Ha, immediately suggests the Hubbard physics character of this problem: as the localization energy cost becomes large, the energetics of lattice distortions which favor increasing orbital overlap and reduced band energy may lead to structural phase transitions.

Notice also that the corresponding charge distributions will assume even a partially ionic form while preserving an essential itinerant or metallic character. Re-introduction of the Coulomb interactions is not expected to significantly modify the density profile, however the approach will lead to a quite different viewpoint on the character of the effective ionion interactions. The latter should adopt a somewhat longer ranged form than may be expected from linear screening arguments in the NFE limit. This has obvious implications for collective excitations and also for ionic dynamics (the physical character of the liquid state and melting problem, for example). The behavior of the electronic density also sheds light on the structural phase transitions which occur at high pressure: the boundary conditions in the FCC lattice lead to a depletion of the density at the tetrahedral sites, thus reducing the effective available volume for the electrons beyond what is already taken away by the impenetrable spheres: a structural phase transition to a state which avoids such depleted small enclosures and allows the electron liquid to reduce its zero-point energy by fully occuping available space thus may become energetically competitive at high pressure. In particular, planar structures allowing the aggregation of the itinerant charge in effectively 
two dimensional NFE gases in planar interstices may become favored. The optical response is also anticipated to be unlike that of NFE-based systems, though a Drude edge should be preserved. Because of band narrowing, the density of states at the Fermi level could be high, and the possibility within a dynamic ionic environment of electron pairing may be enhanced. Some of these features as well as mixtures and complex structures are presently under examination.

This work was supported by the National Science Foundation under grant DMR-0601461. B. Rousseau would like to acknowledge fruitful discussions with S. Gravel and Professor S. Bonev.

* br75@cornell.edu

[1] E. Wigner and F. Seitz, Phys. Rev. 43, 804 (1933), URL http://link.aps.org/abstract/ $\mathrm{PR} / \mathrm{v} 43 / \mathrm{p} 804$.

[2] E. Wigner and F. Seitz, Phys. Rev. 46, 509 (1934), URL http://link.aps.org/abstract/ $\mathrm{PR} / \mathrm{v} 46 / \mathrm{p} 509$.

[3] V. F. E.G. Maksimov, M.V. Magnitskaya, Physics- Uspekhi 48, 761 (2005).

[4] H. Katzke and P. Toledano, Physical Review B 71, 184101 (2005), URL http://link.aps. org/abstract/PRB/v71/e184101.

[5] J. B. Neaton and N. W. Ashcroft, Nature 400, 141 (1999).

[6] J. B. Neaton and N. W. Ashcroft, Phys. Rev. Lett. 86, 2830 (2001).

[7] Y. B. M. Yu. A. Kuperin, S.B. Levin and B. Pavlov, Computers Math. Applic. 34, 599 (1997).

[8] D. J. Chadi and M. L. Cohen, Phys. Rev. B 8, 5747 (1973).

[9] J. Rogan and M. Lagos, Phil. Mag. B 81, 551 (2001).

[10] The goal of this work is not to produce quantitatively precise results but rather to observe the trends in the model and use them as insight to understand the behavior of the simple metals under pressure. "Reasonable convergence" means that results did not qualitatively change with increase of the convergence parameters.

[11] We note however that it is difficult to resolve whether this feature is to be expected for the genuinely impenetrable spheres system or if it is an artefact of the finite $V_{0}$, finite basis size approximation. 
[12] F. C. von der Lage and H. A. Bethe, Phys. Rev. 71, 612 (1947).

[13] The boundary conditions were accommodated using an expansion in 3 and 4 (4 and 6) Kubic harmonics for the $\alpha(\gamma)$ representation. The boundary conditions where applied as described in [12]. 